\title{
RASS-SDSS galaxy cluster survey. A ubiquitous dwarf galaxy population in clusters
}

\author{
P. Popesso ${ }^{1}$, A. Biviano ${ }^{2}$, H. Böhringer ${ }^{3}$ \\ and M. Romaniello ${ }^{1}$ \\ ${ }^{1}$ European Southern Observatory, Karl Scharzschild Strasse 2, D-85748 \\ email:ppopesso@eso.org \\ ${ }^{2}$ Osservatorio Astronomico di Trieste, via G. B. Tiepolo 11, I-34131, Trieste, Italy \\ ${ }^{3}$ Max-Planck-Institut fur extraterrestrische Physik, 85748 Garching, Germany
}

\begin{abstract}
We analyze the Luminosity Functions (LFs) of a subsample of 69 clusters from the RASS-SDSS galaxy cluster catalog. The cluster LFs are computed within the physical sizes of the galaxy systems, $r_{500}$ and $r_{200}$. When calculated within the cluster physical sizes, all the cluster LFs appear to have the same shape, well fitted by a composite of two Schechter functions with a marked upturn and a steepening at the faint-end.

We determine the composite LF for early- and late-type galaxies, where the typing is based on the galaxy $u-r$ colors. The late-type LF is well fitted by a single Schechter function with a steep slope. The early-type LF instead cannot be fitted by a single Schechter function, and a composite of two Schechter functions is needed. The faint-end upturn of the global cluster LF is due to the early-type cluster galaxies. The shape of the bright-end tail of the early-type LF does not seem to depend upon the local galaxy density or the distance from the cluster center. The late-type LF shows a significant variation only very near the cluster center. On the other hand, the faint-end tail of the early-type LF shows a significant and continuous variation with the environment.
\end{abstract}

Keywords. galaxies: fundamental parameters, galaxies: clusters: general, galaxies: dwarf

\section{Introduction}

The galaxy Luminosity Function (LF) is a fundamental tool for understanding galaxy evolution and faint galaxy populations. The shape of the cluster LF provides information on the initial formation and subsequent evolution of galaxies in clusters while the slope of the faint-end indicates how steeply the dwarf number counts rise as a function of magnitude.

Much work has been done on the cluster LF, with various groups finding differences in its shape and the faint-end slope. Whether the LF of cluster galaxies is universal or not, and whether it is different from the LF of field galaxies are still debated issues. Moreover, the LF of cluster galaxies is typically observed to steepen faint-ward of $M_{g} \sim-18$, with power-law slopes $\alpha \sim-1.8 \pm 0.4$. This corresponds to the debated upturn of the cluster $\mathrm{LF}$ due to an excess of dwarf galaxies relative to the field LF.

In this work we use SDSS data (Abazajian et al. 2003) to show that, when measured within the physical size of the system, given by either $r_{200}$ or $r_{500}$, the LF is invariant for all clusters, both at the bright and at the faint end. We also investigate the nature of the dwarf galaxies in clusters by studying their color distribution. The analysis is performed 

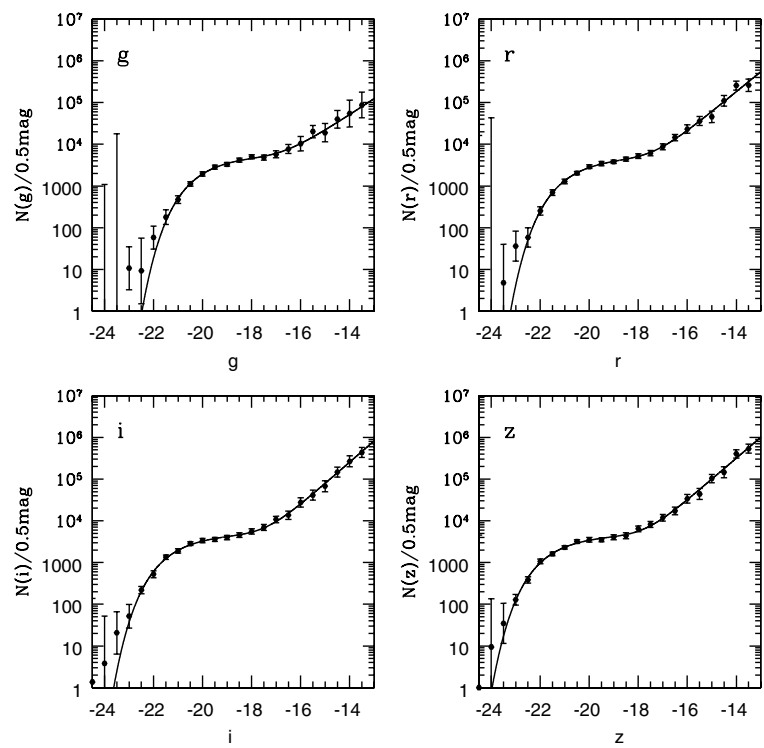

Figure 1. The 4 panels show the composite LFs in the 4 Sloan bands. The individual LFs used to calculate the composite LFs are measured within the physical sizes of the clusters, as given by $r_{200}$.
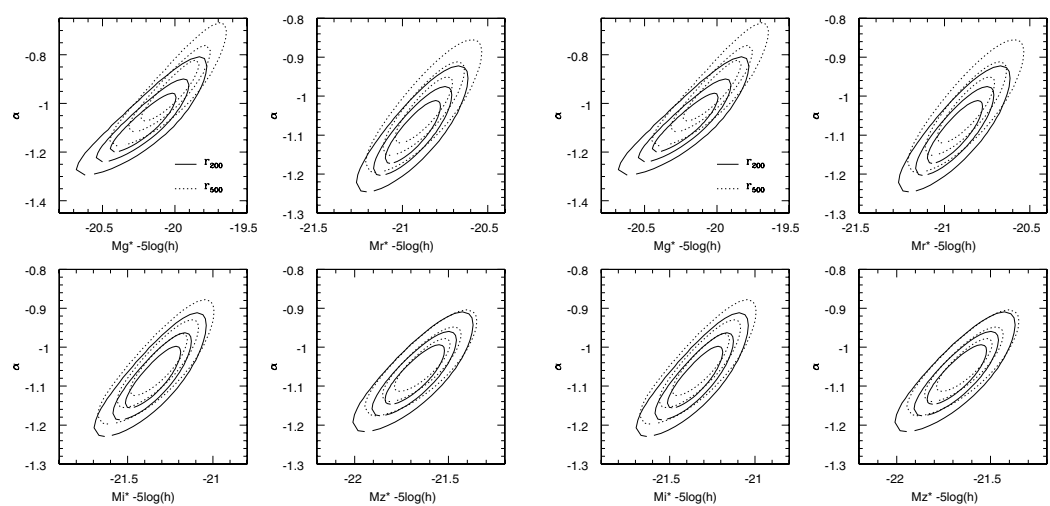

Figure 2. Contour plots of the $68 \%, 95 \%$, and $99 \%$ confidence levels of the parameters of the bright-end component (4 panels on the left-side) and faint-end component (4 panels on the right-side) of the double-Schechter function fit to the 4 SDSS bands composite LFs. Solid (dotted) contours show the results for the composite LF computed within $r_{200}$ (respectively $\left.r_{500}\right)$.

on a subsample of 69 clusters taken from RASS-SDSS galaxy cluster sample (Popesso et al. 2004, 2005).

\section{The Cluster Luminosity Function}

The composite LF is obtained by stacking the individual cluster LFs estimated within $r_{200}$, according to the prescription of Colless (1989). Fig. 1 shows the cluster LF in four SDSS photometric bands. In all the bands, a single Schechter function does not provide 

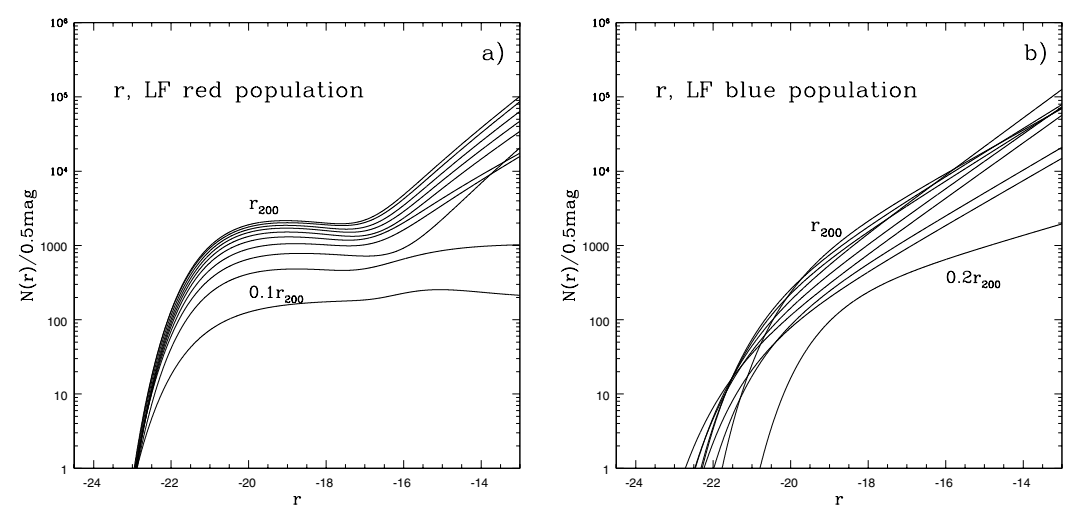

Figure 3. The cluster LFs within different cluster apertures in the $r$ band per morphological type. The increment of the apertures is $0.1 \times r_{200}$. The normalization of the fitting function is increasing at larger apertures. Panel $a$ ) shows the LF of the cluster red galaxy population, calculated within different clustercentric apertures expressed in unit of $r_{200}$. Panel $b$ ) shows the same for the cluster blue galaxy population. For simplicity we only plot the best fitting functions and not the data points.

acceptable fits, and a composite of two Schechter functions is needed. The confidence-level contours of the best-fit parameters of the bright- and faint-end Schechter components are shown in Figs. 2. Both results for the composite LF within $r_{500}$ (dotted contours) and $r_{200}$ (solid contours) are shown. Clearly, the best-fit Schechter function to the LF bright-end does not change significantly from $r_{500}$ to $r_{200}$ (see the 4 left-side panels of the figure). However, the faint-end LF steepens significantly (by $0.1-0.15$ dex) from $r_{500}$ to $r_{200}$, and the characteristic magnitude correspondingly brightens by $0.3-0.4$ magnitudes (see the 4 right-side panel of the figure), thereby indicating an increasing DGR with radius. Moreover, with a simple $\chi^{2}$ test we check the agreement between the composite and individual LFs. For $90 \%$ of the clusters the probability that the composite and individual LFs are drawn from the same parent distribution is larger than $95 \%$. Therefore, we conclude that, when measured within the physical size of the systems, the cluster LF is universal.

\section{The Cluster LF per galaxy type and the environmental dependence}

Using a color cut (Strateva et al. 2001) we separate early type from late type galaxies and check which type of galaxies is responsible for the bimodal behavior of the cluster LF. Moreover, in order to gain insight into the processes responsible for the shaping of the LF in clusters, we here examine also the dependence of the LF on the environmental conditions. In particular we analyze how the LF shape, and the relative fraction of red and blue dwarf galaxies, vary as a function of the clustercentric distance. Fig. 3 shows the behavior of the cluster LF calculated within different clustercentric apertures, separately for the early-type (panel $a$ ) and late-type (panel $b$ ) galaxy populations. Distances are in units of $r_{200}$. For simplicity we only plot the best fitting functions and not the data-points. The early-type LF is close to a Schechter function at the center of the cluster (within $\left.0.2 r_{200}\right)$ and shows a marked upturn after-wards. The location of the upturn varies from $-16.2 \pm 0.3 \mathrm{mag}$ at distances $\leqslant 0.3 r_{200}$ to $-17.4 \pm 0.4$ at distances $\leqslant r_{200}$. The late-type $\mathrm{LF}$ is well fitted by a single Schechter function at any clustercentric distance. Therefore, 

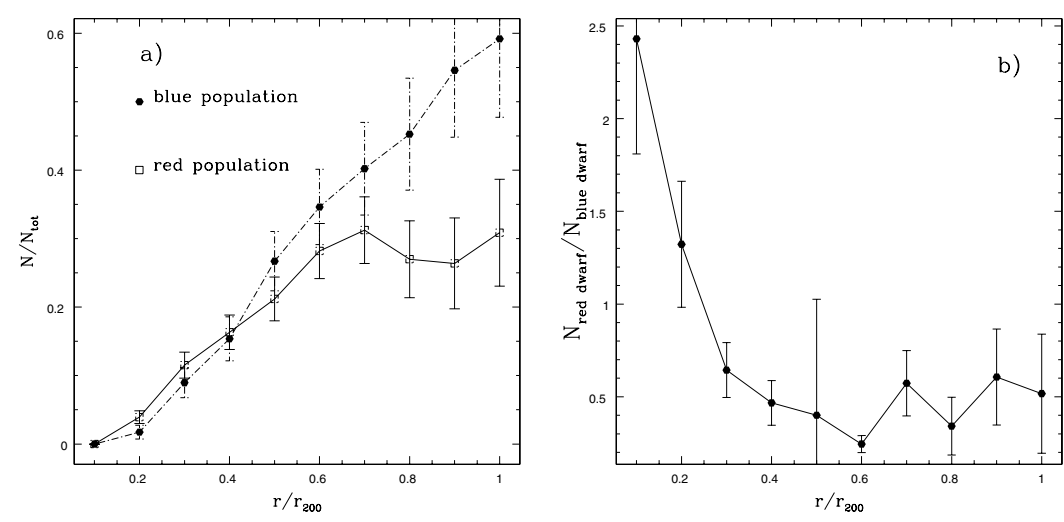

Figure 4. The fraction of red and blue dwarf galaxies as a function of the cluster environments. Panel $a$ ) shows the cumulative radial profile of the fraction of blue (filled points) and red (empty squared) dwarf galaxies $\left(-18 \leqslant M_{r} \leqslant-15 \mathrm{mag}\right)$. The fraction is defined on the total number of cluster dwarf galaxies in the considered magnitude range. Panel $b$ ) shows the differential radial profile of the ratio between red and blue dwarf galaxies.

we conclude that the dwarf early type galaxies are responsible for the upturn and the steepening at the faint-end of the cluster LF.

In order to quantify the relative contribution of the early- and late-type dwarf galaxy populations to the faint end of the LF, and its dependence on the environment, we analyse the radial (cumulative and differential) profile of the dwarf systems in the clusters. The cumulative profiles of the fractions of dwarf galaxies of both the early- and the late-type are shown in Fig. $4 a$. The center $\left(\leqslant 0.4 r_{200}\right)$ contains less than $30 \%$ of dwarf galaxies (half of them are red systems), in the selected magnitude range. Dwarf galaxies are more abundant in the cluster outskirts; the high-density environment in the cluster cores is hostile to dwarf galaxies. The early-type dwarf galaxies represents $35 \%$ of the whole dwarf population within $r_{200}$, i.e. most of the dwarf galaxies are of late-type. However, the dwarf early-type galaxies are the dominant dwarf population in the region within $0.4 r_{200}$, their relative fraction reaching a plateau at $\simeq 0.6 r_{200}$, while the late-type dwarf galaxies are more abundant in clusters outskirts.

\section{Conclusion}

We can draw the following conclusions from our observational results. First, the universality of the cluster LF suggests that whatever shapes the cluster LF is not strictly dependent on the cluster properties. Second, the difference between the cluster and field LF seems to be related to an excess of dwarf early-type galaxies in clusters. Hence, there is a cluster-related process that leads to the formation of dwarf early-type galaxies, regardless of the cluster intrinsic properties. The process cannot be related, e.g., to the intra-cluster gas density, or the cluster velocity dispersion, or the cluster mass, hence, a process like ram-pressure would seem to be ruled out. We suggest as alternative solution the 'harassment' scenario proposed by Moore et al. $(1996,1998)$. In this scenario, close, rapid encounters between galaxies can lead to a radical transformation of a galaxy morphology. Gas and stars are progressively stripped out of the disk systems, eventually leaving a spheroidal remnant, that resembles an S0 galaxy or a dwarf spheroidal, depending on the size of the progenitor. 


\section{References}

Abazajian, K., Adelman-McCarthy, J., Agüeros, M., et al. 2003, AJ 126, 2081

Colless, M. 1989, MNRAS 237, 799

Moore, B., Katz, N., Lake, G., Dressler, A. \& Oemler, A., Jr. 1996, Nature 379, 613

Moore, B., Lake, G. \& Katz, N. 1998, ApJ 495, 139

Popesso, P., Böhringer, H., Brinkmann J., Voges, W. \& York, D. G. 2004b, A\&A A 423, 449

Popesso, P., Biviano, A., Böhringer, Romaniello, M. \& Voges, W. 2004c, A\&GA 433, 431

Strateva, I., Ivezić, Z., Knapp, G., et al. 2001 AJ 122, 1861

\section{Discussion}

GALLAGHER: It would be interesting to estimate the possible rate of evolution of the red dwarf population. One rough way to estimate this would be to compare the number of red dwarfs to blue dwarfs that are in transition to becoming red. Is it possible to do this from your data?

Popesso: We are trying to estimate a transition rate by studying the "upturn galaxies". They are objects in the magnitude range $-19<M_{r}<16$ mag, very concentrated but in star formation. We are investigating whether they are the galaxy transition type between the blue and the red dwarf galaxies. This analysis, in addition to the ratio of the number of red and blue dwarf systems in the individual clusters, would help in understanding the transition process.

Moore: Are the faint end slopes you measure consistent with $-1.2,-1.3$ measured in the Fornax, Virgo clusters?

Popesso: Our faint end has a slope of -2 in the 4 Sloan bands (g, r, i, z), therefore it is not consistent with the one observed in Fornax and Virgo. Nevertheless we think that the discrepancy is due to an aperture effect. We have shown that the slope of the cluster LF strongly depends on the environmental conditions - the faint end of the central cluster region is much flatter than the faint end in the cluster outskirts.

ZibetTi: A concern about the universality of the LF: From the example plots it seems that there are some clusters where the faint end is much less steep than in the stacked LF. Can we still speak of a universal LF? Have you tried bootstrapping your sample to estimate the cluster-to-cluster variation of the faint end?

Popesso: For some objects the composite LF is not a very good representation of the individual cluster LF. We have checked that for more than $90 \%$ of those systems there are projection effects due to a galaxy overdensity at higher redshift along the same line of sight. Moreover, the distribution of the DGR has a small dispersion, therefore the faint end seems to have the same slope in all the clusters. We did not yet apply bootstrapping yet. 